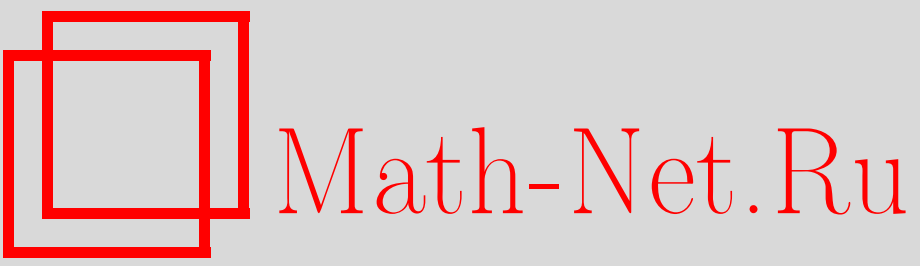

В. И. Субботин, Об одном классе многогранников с симметричными звездами вершин, Итоги науки и техн. Сер. Соврем. мат. и ее прил. Темат. обз., 2019, том 169, 88-97

DOI: https://doi.org/10.36535/0233-6723-2019-169-88-97

Использование Общероссийского математического портала Math-Net.Ru подразумевает, что вы прочитали и согласны с пользовательским соглашением

http://www. mathnet.ru/rus/agreement

Параметры загрузки:

IP: 54.224 .135 .184

26 апреля 2023 г., 13:33:21 


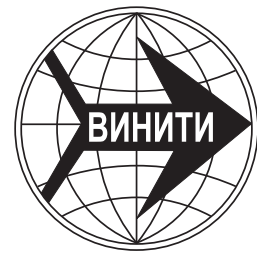

ИТОГИ НАУКИ И ТЕХНИКИ.

Современная математика и ее приложения.

Тематические обзоры.

Том 169 (2019). С. 88-97

DOI: 10.36535/0233-6723-2019-169-88-97

УДК 514.172 .45

\title{
ОБ ОДНОМ КЛАССЕ МНОГОГРАННИКОВ С СИММЕТРИЧНЫМИ ЗВЕЗДАМИ ВЕРШИН
}

\author{
(c) 2019 г. $\quad$ В. И. СУББОТИН
}

\begin{abstract}
АннотАция. Рассмотрено влияние локальной симметрии звезд некоторых вершин замкнутого выпуклого многогранника в $E^{3}$ на его геометрию. Доказана теорема о полной классификации симметричных многогранников, у которых некоторые вершины имеют симметричные звезды из дельтоидных или ромбических граней. В доказательстве использованы ранее введенные автором так называемые сильно симметричные многогранники и лемма о локальных условиях симметрии.
\end{abstract}

Ключевые слова: выпуклый многогранник, звезда вершины, сильно симметричный многогранник, $F S$-многогранник, дельтоидная вершина, ромбическая вершина, $R D S$-многогранник.

\section{ON A CLASS OF POLYHEDRA WITH SYMMETRICAL VERTEX STARS}

\author{
(C) 2019 V. I. SUBBOTIN
}

\begin{abstract}
The influence of the local symmetry of stars of some vertices of a closed convex polyhedron in $E^{3}$ on its geometry is considered. A theorem on the complete classification of symmetric polyhedra some of whose vertices possess symmetric stars od deltoid or rhombic faces is proved. In the proof, we use so-called strongly symmetric polyhedra and the lemma on local symmetry conditions previously introduced by the author.
\end{abstract}

Keywords and phrases: convex polyhedron, vertex star, strongly symmetric polyhedron, FSpolyhedron, deltoid vertex, rhombic vertex, $R D S$-polyhedron.

AMS Subject Classification: 52B10, 52B15

1. Введение. В работе рассматриваются замкнутые выпуклые симметричные многогранники в $E^{3}$. Под симметричностью многогранника понимается наличие в нем хотя бы одного нетривиального элемента симметрии. Нас будет интересовать связь условий симметрии, которым подчинены некоторые элементы многогранника, с его геометрией.

Как известно, группа симметрий многогранника не определяет однозначно даже его комбинаторную структуру. Однако, условия симметрии элементов многогранника могут накладывать на его строение некоторые ограничения.

Например, если для некоторого класса $M$ многогранников группа поворотов (с отражениями) транзитивна на вершинах каждого многогранника, то $M$ будет состоять только из изогонов. Изогон - выпуклый многогранник, все многогранные углы которого равны; при этом каждый изогон может быть реализован с сохранением комбинаторного строения в виде равноугольнополуправильного (архимедова) многогранника с правильными гранями (см. $[1,6,8])$. 
В качестве второго примера рассмотрим класс многогранников только с одним условием симметрии, а именно, условием правильности всех граней многогранника. Как известно, получим полностью перечисленный класс правильногранных многогранников Джонсона-Залгаллера, (cм. $[2,11])$.

В настоящей работе нас будут интересовать такие условия симметрии, которые позволяют, хотя бы комбинаторно, описать весь класс удовлетворяющих этим условиям многогранников. Иногда может оказаться, что рассматриваемый класс многогранников содержит в себе класс правильных (платоновых); тогда можно говорить, что этот класс обобщает класс правильных многогранников.

Другие примеры полного описания некоторых классов симметричных многогранников можно найти в $[1,3,5-10,12]$.

Одним из классов с условиями симметрии, все многогранники которого удается найти, является класс многогранников, сильно симметричных относительно вращения, введенных автором в [3]. Согласно определению в [3], сильно симметричный относительно вращения, или «F -многогранник» (от первых букв слов face и symmetry) - это такой замкнутый выпуклый многогранник в $E^{3}$, для каждой грани $F$ которого существует нетривиальная ось вращения многогранника, перпендикулярная $F$ и пересекающая относительную внутренность $F$.

Условия симметрии в определении сильно симметричного относительно вращения граней многогранника предполагают, что каждая ось вращения соответствующей грани является осью вращения всего многогранника. В [4] доказан локальный критерий сильной симметричности, позволяющий заменить условие глобальной симметричности многогранника относительно каждой оси вращения граней локальной симметрией звезд этих граней: для того, чтобы многогранник был $F S$-многогранником, необходимо и достаточно, чтобъ у каждой грани имеласъ нетривиалъная осъ вращения, являющаяся осъю вращения звезды этой грани. Звезда грани $F$, обозначаемая далее $\operatorname{Star}(F)$ - это совокупность грани $F$ и всех граней, имеющих хотя бы одну общую вершину c $F$. В дальнейшем грани с такой локальной осью вращения будем называть локально симметричными.

В [3] найдены все $F S$-многогранники с точностью до комбинаторного строения и с точностью до сохранения направления и порядка осей вращения каждой грани. Среди найденных многогранников есть такие, которые не являются комбинаторно эквивалентными ни правильным, ни полуправильным. Всего имеется семь комбинаторно различных типов таких «неархимедовых» $F S$-многогранников.

В настоящей работе будет рассмотрен один класс симметричных замкнутых выпуклых многогранников в $E^{3}$, у которых существуют симметричные звезды вершин. Под звездой вершины $V$ будем понимать множество $\operatorname{Star}(V)$ граней, имеющих общей вершиной $V$. Симметричность множества $\operatorname{Star}(V)$ означает его вращательную симметрию относительно оси, проходящей через $V$. Целью работы является решение вопроса о влиянии симметрии звезд вершин на геометрию многогранника. Ответ будет дан для случая, важного для приложений, когда симметричное множество $\operatorname{Star}(V)$ состоит из равных дельтоидов или, в частности, ромбов (не квадратов); дельтоид это выпуклый четырехугольник с взаимно перпендикулярными диагоналями, только одна из которых делится другой диагональю пополам. В этом случае оказывается возможным полное перечисление всех симметричных многогранников с таким строением $\operatorname{Star}(V)$ - так называемых $R D S$-многогранников (от первых букв слов rombic, deltoid, symmetry). Примерами $R D S$-многогранников являются один из параллелоэдров - удлиненный ромбододекаэдр (рис. 1(a)) и форма, встречающаяся в кристаллографии (рис. 1(b)).

2. Основные определения. Обозначим $\operatorname{Star}(\operatorname{Star}(V))$ множество всех граней, имеющих хотя бы одну общую вершину с множеством $\operatorname{Star}(V)$, т.е. со звездой вершины $V$. Все оси вращения, встречающиеся далее, считаются нетривиальными.

Определение 1. Грань $F$ многогранника называется локально симметричной, если существует ось вращения $L_{F}$ множества $\operatorname{Star}(F)$, перпендикулярная $F$ и пересекающая относительную внутренность $F$. 


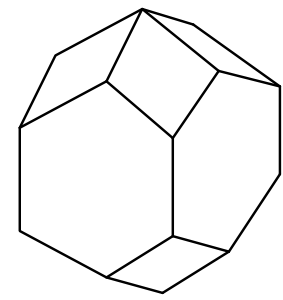

a)

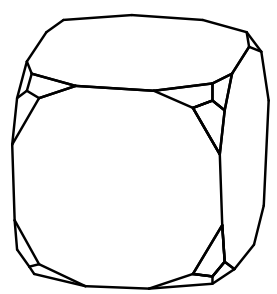

b)

Рис. 1. Примеры $R D S$-многогранников

Определение 2. Вершина $V$ многогранника называется дельтоидной (ромбической), если множество $\operatorname{Star}(V)$ состоит из равных дельтоидов (ромбов), имеющих общей вершиной $V$. Если число дельтоидов (ромбов) в $\operatorname{Star}(V)$ равно $n$, то $V$ называется $n$-дельтоидной $(n$-ромбической).

Определение 3. $n$-Дельтоидная ( $n$-ромбическая) вершина $V$, расположенная на нетривиальной оси вращения $L_{V}$ порядка $n$ множества $\operatorname{Star}(\operatorname{Star}(V))$, называется локально симметричной.

В дальнейшем, если не оговорено специально, рассматриваются только локально симметричные дельтоидные (ромбические) вершины.

Определение 4. Фигура, состоящая из ромбов звезды локально симметричной дельтоидной (ромбической) вершины $V$, называется симметричной дельтоидной (ромбической) шапочкой, а сама вершина $V$ называется вершиной шапочки.

Определение 5. Дельтоидная (ромбическая) вершина называется изолированной, если ее звезда не имеет общих элементов со звездой любой другой дельтоидной (ромбической) вершины многогранника.

Определение 6. Удлинением (вдоль оси вращения) многогранника называется такой сдвиг двух его равных симметричных дельтоидных (ромбических) шапочек вдоль оси вращения, проходящей через вершины этих шапочек, при котором расстояние между вершинами шапочек увеличивается. При этом раздвигаемые общие вершины шапочек, отличные от вершин шапочек, будут концами новых равных и параллельных оси сдвига ребер.

Например, удлиненный ромбододекаэдр получен преобразованием удлинения двух зеркально расположенных 4-ромбических шапочек (рис. 1(а)).

Определение 7. Дельтоидной (ромбической) бипирамидой будем называть многогранник, имеющий две равные зеркально расположенные дельтоидные (ромбические) шапочки, причем вершины дельтоидов (ромбов), противоположные вершинам шапочек, являются общими для обеих шапочек (рис. 2(а)).

Определение 8. Многогранник называется $R D S$-многогранником, если у него существуют локально симметричные дельтоидные (ромбические) вершины $V_{i}$ и существуют грани $F_{k}$, не входящие ни в одну звезду $\operatorname{Star}\left(V_{i}\right)$; при этом каждая грань $F_{k}$ является локально симметричной.

3. Теоремы о $R D S$-многогранниках. Введем понятие дельтоидного преобразования грани многогранника.

Пусть $F$ - некоторая локально симметричная правильная грань многогранника, все вершины которой являются вершинами трехгранных углов $\alpha_{k}$. Пусть плоские углы углов $\alpha_{k}$, отличные от углов грани $F$, равны между собой. Обозначим через $V$ одну из вершин грани $F$, через $A V$ и $V B-$ стороны грани $F$, имеющие общей вершиной $V$, через $U$ - некоторую точку на ребре, составляющем равные углы со сторонами $A V$ и $V B$. Проведем через середины $S$ и $T$ сторон $A V$ и $V B$ и точку $U$ плоскость $\pi$, пересекающую перпендикуляр к центру грани $F$ в некоторой точке $P$. Проделав это построение для всех вершин грани $F$, заключаем в силу локальной симметричности 
грани $F$, что все построенные плоскости пересекутся в точке $P$. По построению, четырехугольник $U S T P$ может быть либо дельтоидом, либо ромбом. Таким образом, вместо грани $F$ получим либо дельтоидную, либо ромбическую шапочку с вершиной $P$. Условимся указанное преобразование грани в дельтоидную или ромбическую шапочку называть дельтоидным преобразованием грани.

Замечание 1. Для четноугольных правильных граней следует учесть возможность следующей небольшой модификации дельтоидного преобразования: плоскость $\pi$, проходящую через точки $S, T, U$ можно провести так, чтобы она прошла через вершины $A, B$ грани. Тогда порядок оси вращения полученной дельтоидной (ромбической) шапочки, очевидно, уменьшится в два раза.

Замечание 2. В результате дельтоидного преобразовании грани не всегда возможно получить дельтоидную шапочку и не всегда - ромбическую, даже варьируя при этом положение точки $U$ вдоль ребра. Именно, если отрезок $U V$ составляет прямые углы с отрезками $A V$ и $V B$, а грань $F$ является квадратом, то дельтоидное преобразование грани даст только ромбическую шапочку, даже при изменении положения точки $U$ на ребре. В этом случае для неквадратной правильной грани получим только дельтоидную шапочку.

Если отрезок $U V$ составляет не прямые углы с отрезками $A V$ и $V B$, то в случае квадратной и треугольной грани в результате дельтоидного преобразования грани получим дельтоидную шапочку, а для других правильных граней в этом случае возможна как ромбическая шапочка, так и дельтоидная.

Эти факты легко понять, если учесть, что форма четырехугольника USTP зависит от того, равны или нет между собой треугольники $U S T$ и $S T P$.

Теорема 1. Для каждого $R D S$-многогранника $M$ существует такой многогранник $M^{\prime}$, сильно симметричный относительно вращения граней, что $M$ может быть получен из $M^{\prime}$ дельтоидным преобразованием некоторых граней.

Доказательство. Разобьем доказательство на две части. Сначала рассмотрим случай, когда у $R D S$-многогранника дельтоидные (ромбические) вершины изолированные, а во второй части когда эти вершины неизолированные, т.е. шапочки имеют общие точки.

1. Итак, пусть дан $R D S$-многогранник $M$ с изолированными дельтоидными (ромбическими) вершинами. Рассмотрим одну из таких вершин $V$. Проведем отсечение вершины $V$ плоскостью $\delta$, перпендикулярной оси вращения, проходящей через $V$ (определение отсечения вершины см. в [13]). Отсечение проведем так, чтобы плоскость $\delta$ прошла через диагонали всех дельтоидов (ромбов) шапочки с вершиной $V$. В результате отсечения получим правильную грань $F$. Треугольники, оставшиеся от дельтоидов (ромбов) после отсечения, образуют замкнутую цепь граней $C$. Любые два соседних треугольника цепи $C$ имеют по одной общей вершине. Рассмотрим один из треугольников $T \in C$. Рассмотрим три грани $F_{1}, F_{2}, F$, стороны которых образуют треугольник $T$; продолжим эти грани до их пересечения в точке $K$. Точка $K$ будет новой вершиной граней $F_{1}, F_{2}, F$. Если сделать такое построение для всех треугольников цепи $C$ и всех дельтоидных (ромбических) вершин многогранника $M$, то грани $F_{1}, F_{2}, F$ останутся локально симметричными, и мы получим новый многогранник $M^{\prime}$, принадлежащий классу $F S$.

Если провести теперь все построения в обратном порядке, то из $F S$-многогранника $M^{\prime}$ получим $R D S$-многогранник $M$.

2. Пусть $R D S$-многогранник $M$ является многогранником с неизолированными дельтоидными (ромбическими) вершинами. Согласно определению $R D S$-многогранника, все грани, не входящие в звезду некоторой дельтоидной (ромбической) вершины, и все дельтоидные (ромбические) шапочки являются локально симметричными. Поэтому мы можем применить локальный критерий сильной симметричности из [4], где каждая грань предполагалась локально симметричной. Действительно, для того, чтобы применить этот критерий, достаточно в рассуждениях при доказательстве в [4] заменить только некоторые локально симметричные грани на локально симметричные шапочки, и доказательство проводится дословно так же. Поэтому для $R D S$-многогранника $M$ с неизолированными дельтоидными (ромбическими) вершинами существует $F S$ многогранник $L$ с теми же осями вращения (с сохранением направления и порядка оси вращения), 
что у многогранника $M$. Применим дельтоидное преобразование к тем граням многогранника $L$, которые имеют соответственные оси дельтоидных (ромбических) шапочек в многограннике $M$. Получим в $L$ дельтоидные (ромбические) шапочки, соответствующие шапочкам в $M$. Следовательно, дельтоиды (ромбы) различных шапочек многогранника $M$ могут иметь общими только по одной точке, а именно вершине, противоположной вершинам шапочек. Теорема 1 доказана.

В следующей теореме найден весь класс $R D S$-многогранников.

Теорема 2. Класс RDS содержит только следующие многогранники:

(1) две бесконечных серии «бипирамид» с двумя n-дельтоидными вериинами, $n=3,5,6,7, \ldots$;

(2) тридиать шесть комбинаторно различных типов многогранников с дельтоидными вериинами;

(3) девять комбинаторно различных типов многогранников с ромбическими вершинами;

(4) два комбинаторно различных 150-гранника, содержащих как дельтоидные, так и ромбические вершины.

Доказательство. Воспользуемся теоремой 1 , из которой следует, что для нахождения всех $R D S$ многогранников достаточно выбрать из всего класса $F S$-многогранников те, которые допускают дельтоидное преобразование граней. Таким образом, нам понадобятся только $F S$-многогранники, у которых имеются правильные грани с трехгранными вершинами.

Если иметь в виду только комбинаторный тип, то кратко результат [3] о нахождении всех $F S$-многогранников состоит в том, что каждый из $F S$-многогранников комбинаторно эквивалентен либо правильному многограннику, либо равноугольно-полуправильному (архимедову), либо двойственному к равноугольно-полуправильному, либо имеет один из семи комбинаторных типов, отличный от типа многогранника любого из перечисленных классов. При этом не каждый равноугольно-полуправильный и не каждый двойственный к равноугольно-полуправильному комбинаторно эквивалентен некоторому $F S$-многограннику. Упомянутые семь комбинаторных типов «неархимедовых» $F S$-многогранников следующие (см. [3]):

(i) первый полуусеченный ромбододекаэдр, второй полуусеченный ромбододекаэдр и усеченный ромбододекаэдр получены соответственно отсечением трехгранных, четырехгранных, всех вершин ромбододекаэдра;

(ii) первый полуусеченный ромботриаконтаэдр, второй полуусеченный ромботриаконтаэдр и усеченный ромботриаконтаэдр получены соответственно отсечением, трехгранных, пятигранных и всех вершин ромботриаконтаэдра;

(iii) седьмой «неархимедов» $F S$-многогранник - полуусеченный куб - получен отсечением вершин куба через одну.

Для доказательства теоремы 2 сначала найдем все $R D S$-многогранники с изолированными дельтоидными вершинами. На рис. 2 представлены фрагменты схем строения таких многогранников. Цифрами на этом рисунке указаны порядки осей вращения некоторых локально симметричных недельтоидных граней.

Для бесконечной серии прямых призм с правильными неквадратными основаниями, очевидно, справедливо следующее: дельтоидное преобразование их оснований приводит к бесконечной серии «дельтоидных бипирамид». Одна из этих бипирамид изображена на рис. 2(а). Прямая призма с квадратным основанием пока не рассматривается, так как дельтоидное преобразование ее оснований дает только удлиненный ромбододекаэдр с двумя 4-ромбическими вершинами.

На рис. 2(b) изображен фрагмент схемы архимедова многогранника - усеченного тетраэдра.

Укажем многогранники, из которых получены многогранники, фрагменты схем которых представлены на рис. 2:

(c) - усеченный октаэдр;

(d) - усеченный икосаэдр;

(e) - усеченный куб;

(f) - усеченный додекаэдр;

(g) - полуусеченный куб;

(h) - дважды усеченный куб; 
ОБ ОДНОМ КЛАССЕ МНОГОГРАННИКОВ

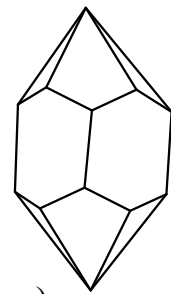

a)

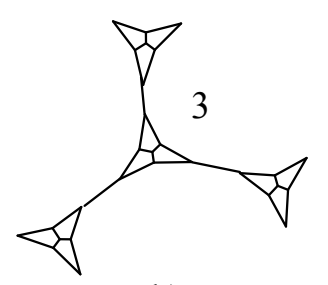

b)

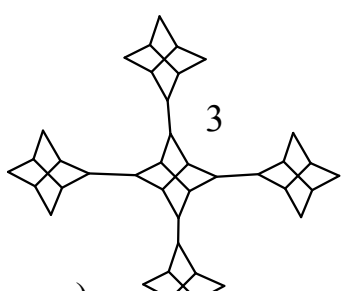

c)

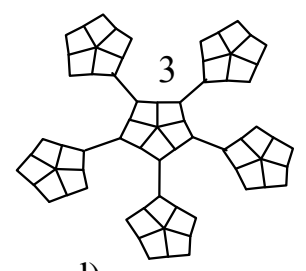

d)

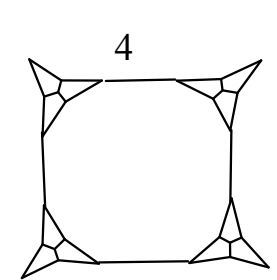

e)

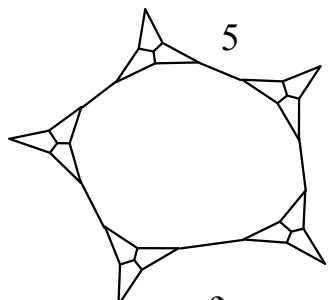

f)
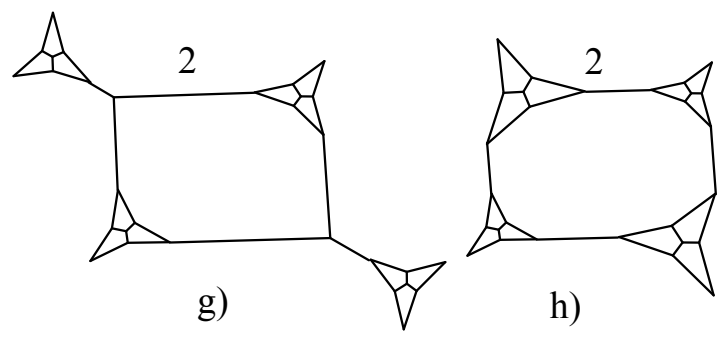

h)

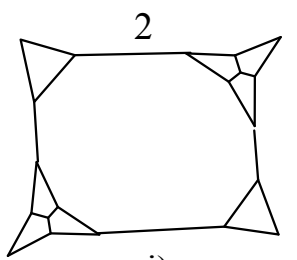

i)

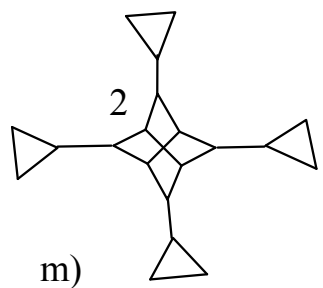

m)

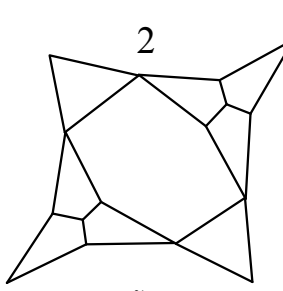

j)

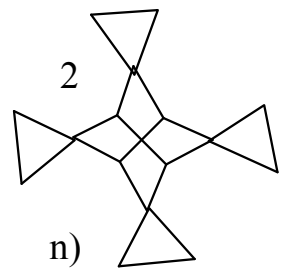

$\mathrm{k})$

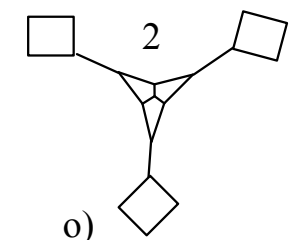

o)
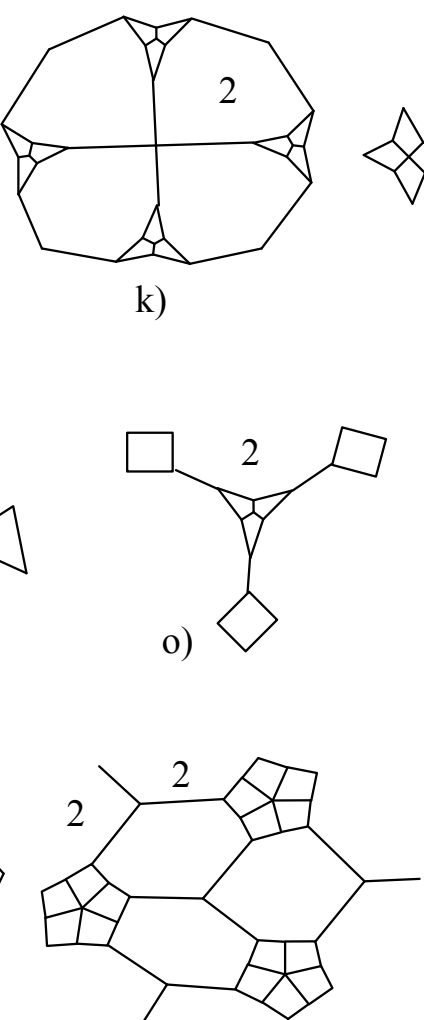

S)

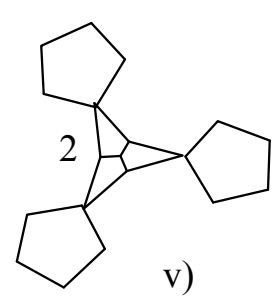

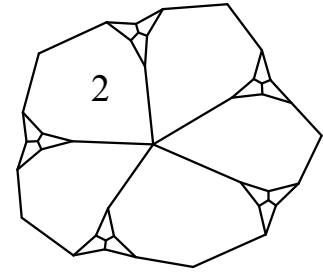

r)

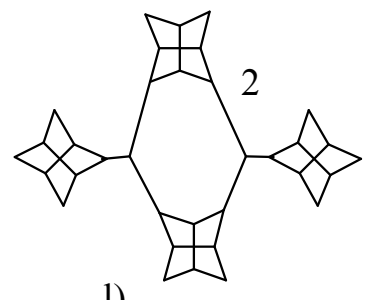

1)

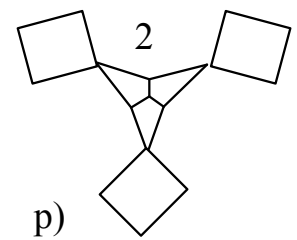

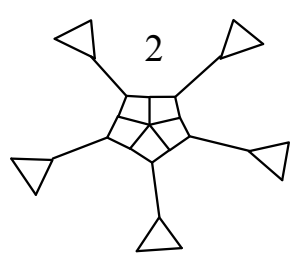

t)

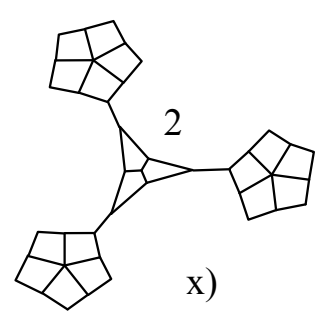

u)

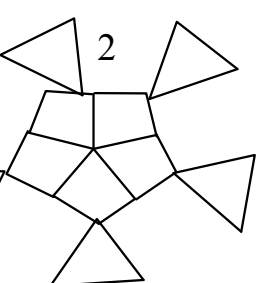

Рис. 2. $R D S$-Многогранники с изолированными дельтоидными вершинами 


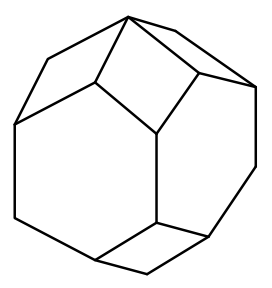

a)

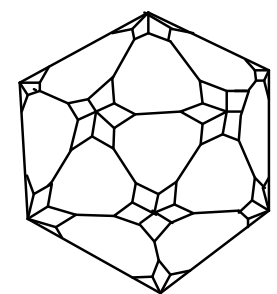

b)

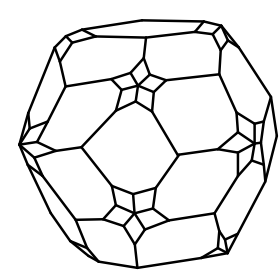

c)

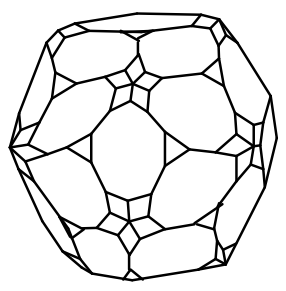

d)

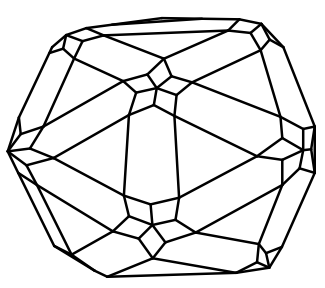

e)

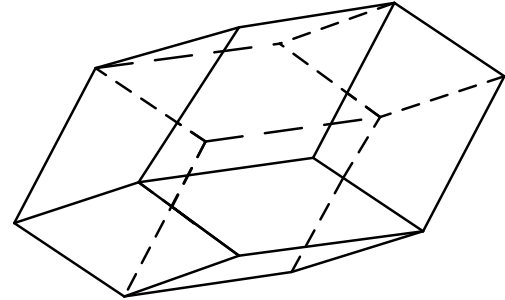

f)

Рис. 3. $R D S$-Многогранники с изолированными ромбическими вершинами

(i), (j) - усеченный куб;

(k) - первый полуусеченный ромбододекадр;

(l) - второй полуусеченный ромбододекаэдр;

(m)-(q) - усеченный ромбододекаэдр;

(r) - первый полуусеченный ромботриаконтаэдр;

(s) - второй полуусеченный ромботриаконтаэдр;

$(\mathrm{t})-(\mathrm{x})$ - усеченный ромботриаконтаэдр.

Введем для каждого из найденных многогранников символ $\{m ; n / p\}$, где $m-$ число граней, $n-$ число $p$-дельтоидных вершин. Тогда многогранники, представленные на рис. 2 , имеют следующие символы:
(a) $\{3 n ; 2 / n\}$,
(g) $\{10 ; 4 / 3\}$;
(h) $\{14 ; 4 / 3\}$;
$n=3,5,6,7, \ldots$;
(i) $\{22 ; 4 / 3\}$;
(j) $\{22 ; 4 / 3\}$;
(c) $\{32 ; 6 / 4\}$;
(k) $\{36 ; 8 / 3\}$;
(e) $\{30 ; 8 / 3\}$;
(l) $\{36 ; 6 / 4\}$;
(f) $\{72 ; 20 / 3\}$;
(m) $\{44 ; 6 / 4\}$;

(n) $\{44 ; 6 / 4\}$;

(o) $\{42 ; 8 / 3\}$;

(u) $\{120 ; 12 / 5\}$;

(p) $\{42 ; 8 / 3\}$;

(q) $\{60 ; 8 / 3 ; 6 / 4\}$;

(r) $\{90 ; 20 / 3\}$;

(s) $\{90 ; 12 / 5\}$;

(t) $\{120 ; 12 / 5\}$;

(v) $\{102 ; 20 / 3\}$;

(w) $\{102 ; 20 / 3\}$;

(x) $\{150 ; 12 / 5 ; 20 / 3\}$.

Найдем теперь все $R D S$-многогранники с изолированными ромбическими вершинами. Для этого необходимо, как следует из замечания 2, применить дельтоидное преобразование к нетреугольным и неквадратным граням тех $F S$-многогранников, у которых такие грани имеют трехгранные вершины. При этом нужно рассмотреть еще те $F S$-многогранники с такими квадратными гранями, все плоские углы трехгранных углов в вершинах которых прямые. Получим многогранники, представленные на рис. 3.

На рис. 3 удлиненный ромбододекаэдр, многогранник (а), есть результат дельтоидного преобразования верхнего и нижнего квадратного оснований прямоугольного параллелепипеда.

Многогранник (b) на рис. 3 получен дельтоидным преобразованием пятиугольных граней усеченного икосаэдра.

Напомним, что второй полуусеченный ромботриаконтаэдр получен из ромботриаконтаэдра отсечением вершин степени 5. Применяя дельтоидное преобразование к его пятиугольным граням, получим многогранник (с) на рис. 3. 


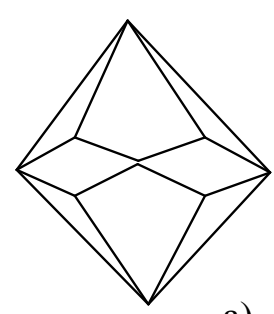

a)

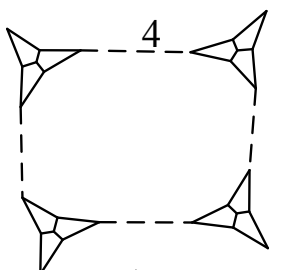

e)

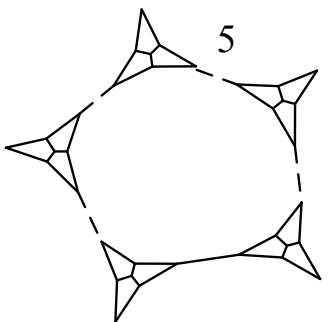

h)

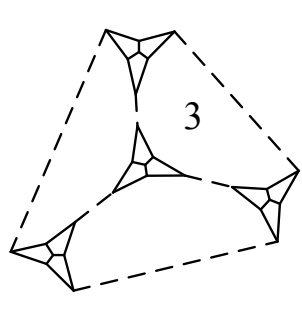

b)

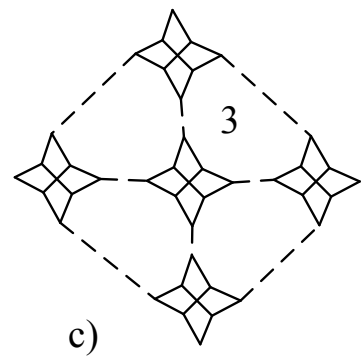

c)

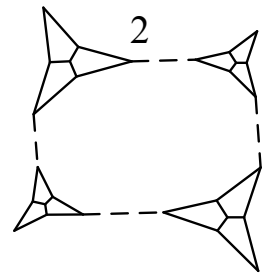

f)

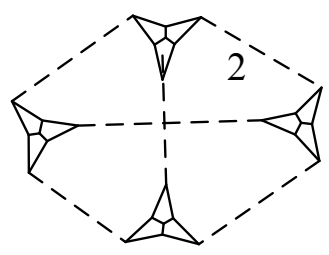

i)

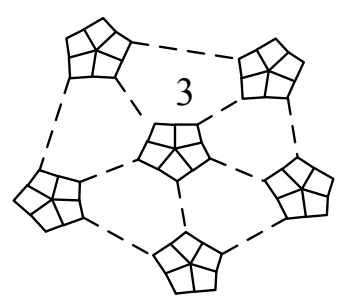

d)<smiles>C1=CC1C1CC2=CC(C2)CC(C2C=C2)C2C=C2C1</smiles>

g)

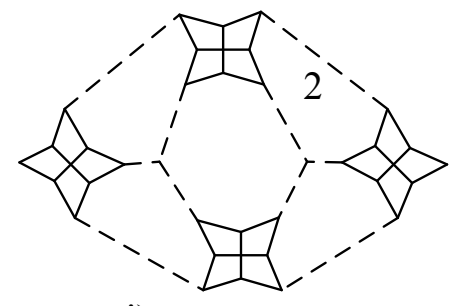

j)

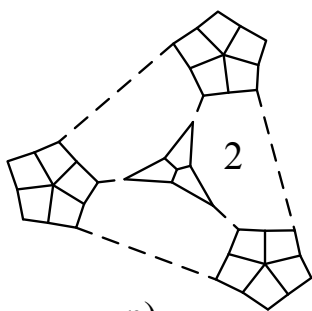

n)

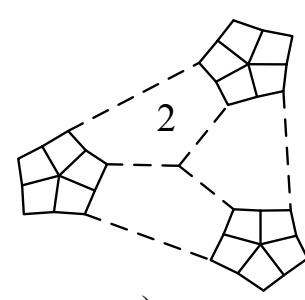

m)

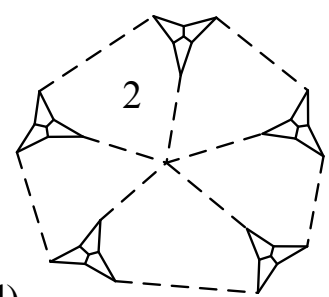

1)

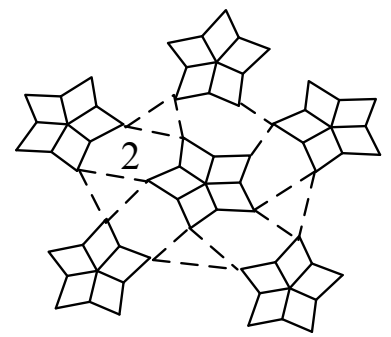

p)

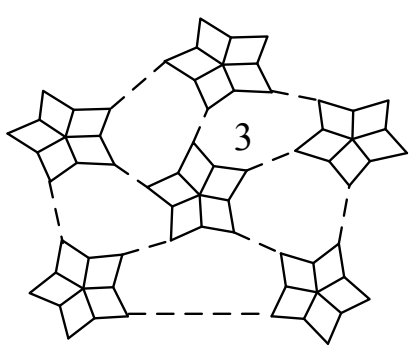

q

Рис. 4. $R D S$-многогранники с неизолированными вершинами 


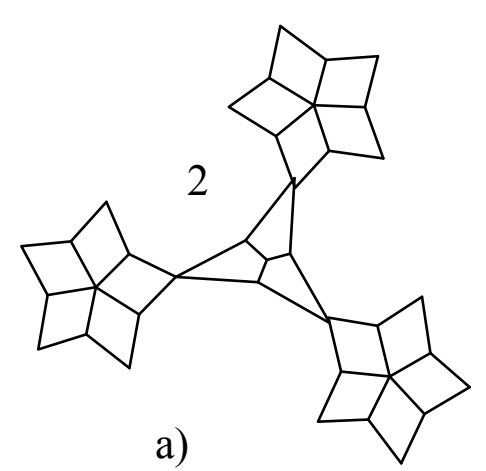

a)

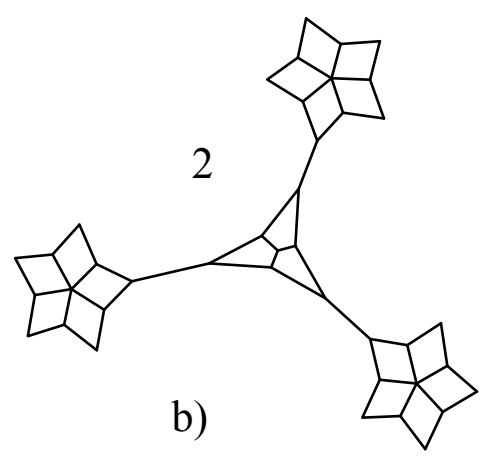

b)

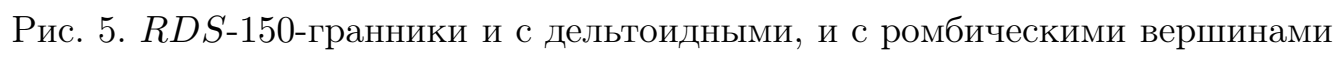

Усеченный ромботриаконтаэдр получен отсечением вершин степени 3 и степени 5 . Применяя дельтоидное преобразование к его пятиугольным граням, получим многогранник (d) на рис. 3. Проводя отсечение вершин степени 3 ромботриаконтаэдра и дельтоидное преобразование таким образом, чтобы треугольные грани многогранника (d) были достаточно большими, получим многогранник (е).

Напомним, что ромбоэдром называется шестигранник с двумя 3-ромбическими вершинами; все его грани являются равными ромбами. Результатом преобразования удлинения, примененного к ромбоэдру, является многогранник (f). Многогранник (f) в нашем случае может быть получен также применением такого дельтоидного преобразования к основаниям правильной шестиугольной призмы, о котором говорилось в замечании 2.

Найдем теперь все многогранники с неизолированными дельтоидными вершинами и все многогранники с неизолированными ромбическими вершинами. Согласно п. 2 доказательства теоремы 1 - это следующие многогранники, фрагменты схем которых изображены на рис. 4 (пунктирной линией указаны отождествленные вершины).

На рис. 4 многогранники (о)-(q) имеют неизолированные ромбические вершины. Применяя дельтоидное преобразование к квадратным основаниям прямоугольного параллелепипеда так, чтобы обе ромбические шапочки имели общие вершины на больших диагоналях ромбов, получим многогранник (о).

Многогранник (р) с неизолированными ромбическим вершинами получен дельтоидным преобразованием достаточно больших пятиугольных граней второго полуусеченного ромботриаконтаэдра.

Многогранник (q) с неизолированными ромбическим вершинами получен дельтоидным преобразованием из усеченного икосаэдра с достаточно большими пятиугольными гранями.

Многогранники (a)-(n) на рис. 4 имеют неизолированные дельтоидные вершины. При этом число граней и число дельтоидных вершин у этих многогранников то же самое, что и у соответствующих им многогранников с изолированными дельтоидными вершинами.

Теперь осталось только проверить, какие из рассмотренных $R D S$-многогранников допускают одновременное наличие и дельтоидных, и ромбических вершин.

$R D S$-Многогогранники и с дельтоидными, и с ромбическими вершинами могут быть получены, очевидно, только из тех $F S$-многогранников, которые имеют более одного типа правильных граней. Рассматривая уже найденные $R D S$-многогранники, видим, что таким многогранником может быть только усеченный ромботриаконтаэдр. Дельтоидным преобразованием его пятиугольных граней ранее получен многогранник, изображенный на рис. 5(d) с 5-ромбическими вершинами. Применяя далее дельтоидное преобразование к его треугольным граням, получим либо многогранник (a), либо (b) на рис. 5.

Таким образом, этими двумя многогранниками исчерпываются все многогранники, которые имеют одновременно и ромбические, и дельтоидные грани.

Мы нашли все $R D S$-многогранники, и теорема 2 доказана. 


\section{СПИСОК ЛИТЕРАТУРЫ}

1. Деза М., Гришухин В. П., Штогрин А. И. Изометрические полиэдральные подграфы в гиперкубах и кубических решетках. - М.: МЦНМО, 2007.

2. Залгаллер В. А. Выпуклые многогранники с правильными гранями// Зап. научн. сем. ЛОМИ. - 1967. - 2. - C. $1-220$.

3. Субботин В. И. Об одном классе сильно симметричных многогранников// Чебышев. сб. - 2016. № 4 . - С. $132-140$.

4. Субботин В. И. О некоторых обобщениях сильно симметричных многогранников// Чебышев. сб. 2015. - № 2. - С. 222-230.

5. Субботин В. И. Сильно симметричные многогранники// Зап. науч. семинаров ПОМИ. $-2003 .-299$. - C. 314-325.

6. Coxeter H. S. M. Regular Polytopes. - N.Y.: Dover, 1973.

7. Coxeter H. S. M. Regular and semi-regular polytopes. III// Math. Z. - 1988. — 200, № 21. — P. 3-45.

8. Cromwell P. R. Polyhedra. - Cambridge: Cambridge Univ. Press, 1999.

9. Farris $S$. L. Completely classifying all vertex-transitive and edge-transitive polyhedra// Geom. Dedic. 1988. - 26, № 1. - P. 111-124.

10. Grunbaum B. Regular polyhedra —old and new// Aequat. Math. — 1977. — 16, № 1-2. - P. 1-20.

11. Johnson N. W. Convex polyhedra with regular faces// Can. J. Math. - 1966. - 18, № 1. - P. 169-200.

12. Wills J. M. On polyhedra with transitivity properties// Discr. Comput. Geom. - 1986. - 1, № 3. P. 195-199.

13. Ziegler G. M. Lectures on Polytopes. - N.Y.: Springer-Verlag, 1995.

Субботин Владимир Иванович

Южно-Российский государственный политехнический университет

им. М. И. Платова, Новочеркасск

E-mail: geometry@mail.ru 